\title{
The Effects of Ripple Characteristics on the Simultaneous Relativistic-Ponderomotive Self-Focusing
}

\author{
M. Moshrelgosha* \\ Department of Physics, University of Qom, P.O. Box 37185-359, Qom, Iran \\ (Received July 28, 2019; revised version October 19, 2019; in final form October 28, 2019)
}

\begin{abstract}
In this study the nonlinear dependences of simultaneous ponderomotive-relativistic self-focusing on the ripple characteristics including the initial width of the ripple, the phase difference between the electric fields of the main beam and the ripple, the amplitude of electric field, and the position of the ripple on the wave front of the beam are found. The intensity profile of the main beam is regarded as Gaussian profile and the ripple is assumed as ring ripple. All the results are verified by solving for the boundary equation of beam width and good agreement is obtained. The presented approach can be applied for other profiles of the main beam and also for other ripple shapes such as the Gaussian ripples.
\end{abstract}

DOI: 10.12693/APhysPolA.136.957

PACS/topics: ring ripple, self-focusing, under-dense plasma

\section{Introduction}

The recent advances in the ultra-short intense lasers and their interaction with plasma have triggered important activities such as laser-driven fusion and monoenergy electron beams $[1,2]$. The mono-energetic electron beams, which are produced in laser-plasma accelerators are the source of important electromagnetic radiations such as $\mathrm{X}$ and gamma rays [3], ultra-short attosecond pulses [4], and terahertz radiation [5]. In the above mentioned applications, as well as all applications of relativistic laser-plasma interaction, the self-focusing phenomena plays an important role, which must be taken into account carefully. By means of this nonlinear effect, the intense pulses can be guided in the plasma medium, over several Rayleigh lengths and without significant energy losses [6-8]. Most of studies have investigated the self-focusing in plasma for beams with smooth profiles of intensity, however, the experimental investigations indicate that the beam intensity profile is not so smooth and the intensity spikes, which are superimposed on the laser beam, can change the intensity profile and the growth of filamentary instability in plasma is important nonlinear phenomenon, which occurs because of the small perturbations in the main beam [9-11]. Variety of studies investigate the growth of different shapes of ripples superimposed on a laser beam such as Gaussian ripple $[12,13]$ and the ring ripple $[14,15]$. Sodha et al. have investigated the dynamics of a radially symmetrical ripple superimposed on a Gaussian laser beam in collisional and collisionless magnetized plasma [16]. In 2007 Sodha sinusoidal instability, superimposed on a uniform electromagnetic beam and the selffocusing of a Gaussian ripple, superimposed on a uniform electromagnetic beam [17]. Misra et al. investigated

\footnotetext{
*e-mail: m.moshkelgosha@qom.ac.ir
}

the ring ripple instability superimposed on a Gaussian electromagnetic beam based on the paraxial-like approximation $[18,19]$. In this approach which is valid in the vicinity of maximum intensity of the ring ripple, the dielectric function, irradiance, and the eikonal are expanded around the maximum of the ring ripple. Following the above mentioned approach Sodha et al. studied the growth of a coaxial ring ripple on a Gaussian electromagnetic beam in the simultaneous presence of ponderomotive and relativistic self-focusing [20]. The simultaneous ponderomotive-relativistic self-focusing happens, when $\tau_{p e}<\tau<\tau_{p i}$. Here, $\tau_{p e}, \tau_{p i}, \tau$ are the electron plasma period, ion plasma period, and the pulse duration, respectively [20-22]. In their study the electric field of the propagating beam is assumed to be composed of the radial electric fields of the Gaussian beam and the ring ripple. This assumption results in the same dielectric function for the ring ripple part as well as the Gaussian part of laser beam and the same focusing factors are obtained for both the main Gaussian beam and the ripple. The authors then presented the governing equation for self-focusing based on the paraxial-like approximation [20].

The above mentioned studies as well as other studies indicate that the ripple parameters affect the focusing of beam considerably [23-26]. However, the effect of ripple parameters on self-focusing is assessed by means of comparing the amount of focusing for few sets of parameters. However, the nonlinear and coupled effect of the involved parameters on self-focusing cannot be founded by the comparison between the focusing rate of some different values and to the author's best knowledge, the accurate dependencies on the ripple parameters have not been presented yet. In this study a simple approach is proposed which provides the precise relations between the rate of focusing and the ripple characteristics. In Sect. 2 the effective focusing parameter (EFP) is introduced. Based on the introduced EFP, the selffocusing dependence on the ring parameters are found in Sect. 3. 


\section{Effective focusing parameter}

The main beam is regarded as the Gaussian beam propagating along $z$-axis with the ring ripple as the small coaxial perturbation. The electric vector of beam is regarded along $y$-axis and the profile of electric field is assumed to be combination of the ripple and main Gaussian beam. The electric field of the Gaussian beam with the coaxial ripple can be expressed [20] as

$$
\boldsymbol{E}=\hat{y} F_{0} \mathrm{e}^{\mathrm{i} \omega t},
$$

where

$\left.F_{0}\right|_{z=0}=E_{00} \mathrm{e}^{-r^{2} / 2 r_{0}^{2}}+E_{10}\left(\frac{r^{2}}{r_{0}^{2}}-\delta\right)^{n / 2} \mathrm{e}^{-r^{2} / 2 r_{1}^{2}} \mathrm{e}^{\mathrm{i} \phi_{p}}$.

Here, $F_{0}$ is the complex amplitude of beam, $E_{00}, E_{10}, r_{0}$, and $r_{1}$ are the initial amplitude of the Gaussian beam, the initial amplitude of the ripple, the initial width of the Gaussian beam, and the initial width of the ripple, respectively. The parameters $n$ and $\delta$ are positive numbers which describe the position of ripple on the wave front of the main beam, $\phi_{p}$ is the initial phase difference between the electric field vectors of the Gaussian and ring ripple and $\omega$ is the angular frequency of beam. The first term on the right hand side of Eq. (2) represents the Gaussian beam and the second term is related to the radial distribution of coaxial perturbation as the ring ripple.

Regarding the simultaneous relativistic-ponderomotive self-focusing, the electron density $N_{e z}$ and the dielectric constant of plasma $\varepsilon$ aremodified [20], according to

$$
\begin{aligned}
& N_{0 z}=N_{e 0}\left[1+\left(\frac{c^{2}}{\omega_{p o}^{2}}\right)\left(\nabla^{2} \gamma-\frac{(\nabla \gamma)^{2}}{\gamma}\right)\right], \\
& \varepsilon(r, z)=1-\Omega_{p}^{2} \frac{N_{0 e}}{N_{0}} .
\end{aligned}
$$

Here, $N_{e 0}$ is as the initial electron density (at $z=0$ ), $c$ is the light speed, $\gamma$ is the relativistic factor, $\omega_{p 0}$ is the angular frequency of plasma, while $\Omega_{p}=\omega_{p e} / \omega$. Based on the paraxial-like approximation the dielectric function of plasma can be expanded [20] as follows

$$
\varepsilon(\chi, z)=\varepsilon_{0}-\chi^{2} \varepsilon_{2},
$$

where

$$
\begin{aligned}
\chi^{2} & =\left[\frac{r^{2}}{r_{0}^{2} f^{2}}-(n+\delta)\right], \\
\varepsilon_{0} & =1-\frac{\Omega_{p 0}^{2}}{\sqrt{1+g_{0}}} \\
& -\frac{2}{\rho^{2} f^{2}}\left[\frac{2 g_{4}(n+\delta)+g_{2}}{1+g_{0}}-\frac{g_{2}^{2}(n+\delta)}{\left(1+g_{0}\right)^{2}}\right], \\
\varepsilon_{2} & =-\frac{g_{2} \Omega_{p 0}^{2}}{2\left(1+g_{0}\right)^{3 / 2}}+\frac{1}{\rho^{2} f^{2}}\left[\frac{12 g_{6}(n+\delta)+8 g_{4}}{1+g_{0}}\right. \\
& \left.-\frac{12 g_{2} g_{4}(n+\delta)+4 g_{2}^{2}}{\left(1+g_{0}\right)^{2}}+\frac{4 g_{2}^{3}(n+\delta)}{\left(1+g_{0}\right)^{3}}\right] .
\end{aligned}
$$

Here $\rho=r_{1} \frac{\omega}{c}$ is the dimensionless parameter of the initial ripple width. The beam width parameter, denoted by $f$, is the ratio of beam width along the propagation with respect to the initial beam width. The coefficients $g_{0}, g_{2}, g_{4}, g_{6}$ are defined as

$$
\begin{aligned}
g_{0} & =\frac{E_{0}^{2}}{f^{2}}\left[\mathrm{e}^{-m(n+\delta)}+p^{2} n^{n} \mathrm{e}^{-(n+\delta)}\right. \\
& \left.+2 p n^{n / 2} \mathrm{e}^{-(m+1)(n+\delta) / 2} \cos \left(\phi_{p}\right)\right], \\
g_{2} & =-\frac{E_{0}^{2}}{f^{2}}\left[m \mathrm{e}^{-m(n+\delta)}\right. \\
& \left.+p m n^{n / 2} \mathrm{e}^{-(m+1)(n+\delta) / 2} \cos \left(\phi_{p}\right)\right], \\
g_{4} & =\frac{E_{0}^{2}}{f^{2}}\left[\frac{m^{2}}{2} \mathrm{e}^{-m(n+\delta)}\right. \\
& \left.-\frac{1}{2 n} p^{2} n^{n} \mathrm{e}^{-(n+\delta)}+p n^{n / 2} \cos \left(\phi_{p}\right)\right], \\
g_{6} & =\frac{E_{0}^{2}}{f^{2}}\left[-\frac{m^{3}}{6} \mathrm{e}^{-m(n+\delta)}+\frac{1}{3 n^{2}} p^{2} n^{n} \mathrm{e}^{-(n+\delta)}\right. \\
& \left.+p n^{n / 2} \mathrm{e}^{-(m+1)(n+\delta) / 2}\left(\frac{m}{4 n}+\frac{1}{3 n^{2}}-\frac{m^{3}}{24}\right) \cos \left(\phi_{p}\right)\right),
\end{aligned}
$$

where $m=\left(\frac{r_{1}}{r_{0}}\right)^{2}$ and $p=\frac{E_{10}}{E_{00}}$.

Using the wave equation and the modification of dielectric constant (Eq. (4)) in the paraxial-like approximation, one can obtain the boundary equation of beam width parameter [20], i.e.,

$$
\begin{gathered}
\frac{\partial^{2} f}{\partial \xi^{2}}=-\frac{1}{2 \varepsilon_{0}} \frac{\mathrm{d} f}{\mathrm{~d} \xi} \frac{\mathrm{d} \varepsilon_{0}}{\mathrm{~d} \xi}+\left[-\frac{2 g_{2}\left(2 g_{0}\left(g_{2}+2 \lambda g_{4}\right)-\lambda g_{2}^{2}\right)}{\varepsilon_{0} g_{0}^{3} f^{3}}\right. \\
\left.+\frac{\left(4 g_{0}\left(2 g_{4}+3 \lambda g_{6}\right)+g_{2}^{2}\right) g_{0}}{\varepsilon_{0} g_{0}^{3} f^{3}}-\frac{\rho^{2} g_{0}^{3} \varepsilon_{2}}{\varepsilon_{0} g_{0}^{3} f}\right]
\end{gathered}
$$

where $\xi=\frac{c}{r^{2} \omega} z$ is the dimensionless propagation length. Equation (8) describes the beam width variation along the propagation axis. Substituting $g_{0}, g_{2}, g_{4}, g_{6}, \varepsilon_{0}$, and $\varepsilon_{2}$ from Eqs. (6), (7) into Eq. (8), it can be seen that beam width parameter is a complicated function of $\Omega_{p}, \rho, n, m, p, \delta$, and $\phi_{p}$. Since the axially homogeneous plasma $\frac{\mathrm{d} \varepsilon_{0}}{\mathrm{~d} \xi}$ is zero, the first term on the right hand side of Eq. (8) vanishes. Therefore this equation reduces to

$$
\begin{gathered}
\frac{\partial^{2} f}{\partial \xi^{2}}-\underbrace{\left(\frac{-2 g_{2}\left(2 g_{0}\left(g_{2}+2 \lambda g_{4}\right)-\lambda g_{2}^{2}\right)}{g_{0}^{3} \varepsilon_{0} f^{3}}\right.}_{\mathrm{EFP}} \\
\underbrace{\left.+\frac{\left(4 g_{0}\left(2 g_{4}+3 \lambda g_{6}\right)+g_{2}^{2}\right) g_{0}}{g_{0}^{3} \varepsilon_{0} f^{3}}-\frac{\rho^{2} g_{0}^{3} \varepsilon_{2}}{g_{0}^{3} \varepsilon_{0} f}\right)}_{\mathrm{EFP}}=0 .
\end{gathered}
$$

In order to solve Eq. (9) the main beam is required initially to be unfocused beam $(f=1)$ and the initial plain wave front should be set at $z=0(\mathrm{~d} / \mathrm{d} z=0)$, because $z=0$ is regarded as the beam waist. The second term in left hand side of Eq. (9) is Effective Focusing Parameter 
(EFP), which comes from two effects. First one is the effect of natural diffraction, which causes the divergence of beam, and the second one is the ponderomotiverelativistic effect, which is responsible for the focusing of beam.

In the differential equations as $\frac{\mathrm{d}^{2} y(t)}{\mathrm{d} t^{2}}+\frac{n_{1}}{y(t)^{n_{2}}}=0$, if $n_{1}$ is negative and $n_{2}$ is positive integer number, the solution for $y(t)$ becomes a diverging function of $t$. This means that $y(t)$ starts to increase with respect to its initial value. In turn, when $n_{1}$ is a positive number, the solution for $y(t)$ becomes a converging (or oscillating) function of $t$. Therefore, when $\partial^{2} f / \partial \xi^{2}$ is positive (focusing condition), then the beam width parameter $f$ will decrease. The beam width will increase (divergence of beam) when $\partial^{2} f / \partial \xi^{2}$ is negative. However, if $\partial^{2} f / \partial \xi^{2}$ is equal to zero, neither convergence nor divergence occurs. The rate of focusing or divergence of beam is related to the dominance of focusing or divergence effects, namely if only the larger positive value of $\partial^{2} f / \partial \xi^{2}$ predicts stronger focusing and the larger negative $\partial^{2} f / \partial \xi^{2}$ refers to more divergence.

Following Moshkelgosha's work [27], this treatment can be applicable for studying the complicated and nonlinear dependence of self-focusing on the involved parameters and one can discuss the behavior of EFP in order to investigate the effect of concerned laser and plasma parameters on rate of beam width reduction. In the next sections, the effect of laser and plasma on self-focusing is discussed based on EFP parameter and the validity of results will be discussed by means of solving for the second order boundary equation of beam width parameter.

\section{The effects of ripple parameters on self-focusing}

In order to understand the effect of involved parameters on self-focusing, the EFP behavior is discussed. The typical and standard set of parameters for the main beam and the ring, is regarded as

$$
\begin{aligned}
& n=1, \quad \alpha E_{0}^{2}=4, \quad \delta=1, \quad \phi_{p}=\frac{\pi}{3}, \quad \Omega_{p 0}^{2}=0.8, \\
& \rho=4, \quad p=0.01, \quad m=0.001, \quad r_{0}=10 \mu \mathrm{m} .
\end{aligned}
$$

The EFP parameter versus the ring characteristics is plotted in Figs. $1-5$ by changing the parameters $\left(n, \delta, \phi_{p}\right.$, $\left.\alpha E_{10}^{2}, m\right)$, one by one, in the acceptable range for each parameter.

The EFP behavior versus $n$ parameter is shown in Fig. 1a. The key aspects of this figure are listed below

- For $0<n<2$ the EFP parameter increases sharply with increasing $n$. Therefore, the stronger focusing of beam is expected by increasing $n$ in this range.

- For $2<n<9$ the inverse dependence of EFP on $n$ is seen, which predicts the weaker focusing for larger $n$ in this region.

- For $n>9$ the value of EFP rises by increasing $n$ which predicts the stronger focusing at larger $n$.
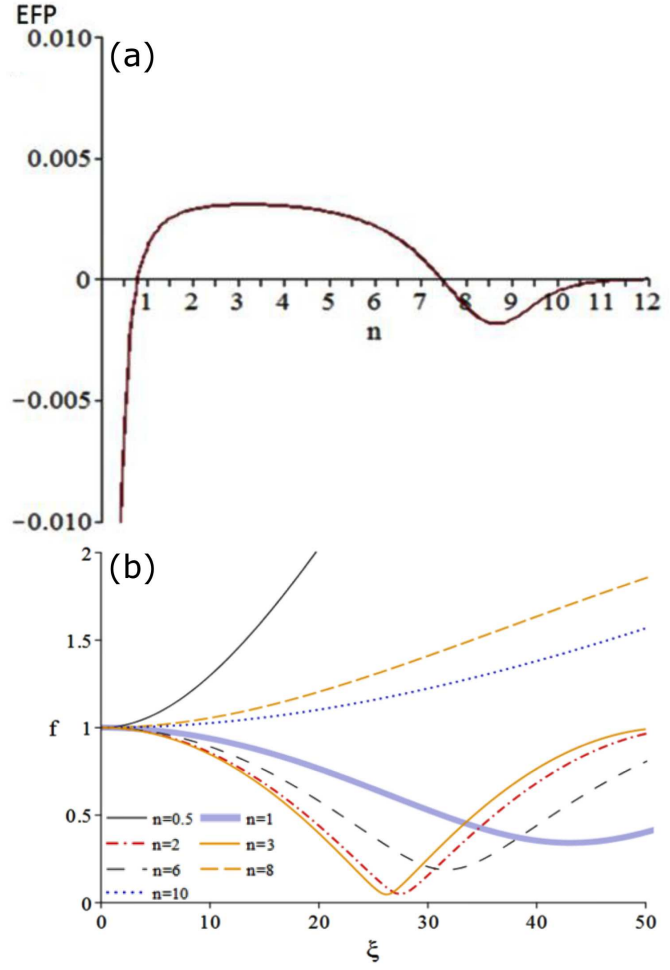

Fig. 1. (a) The introduced EFP parameter versus $n$. (b) The result of solving for self-focusing Eq. (9).
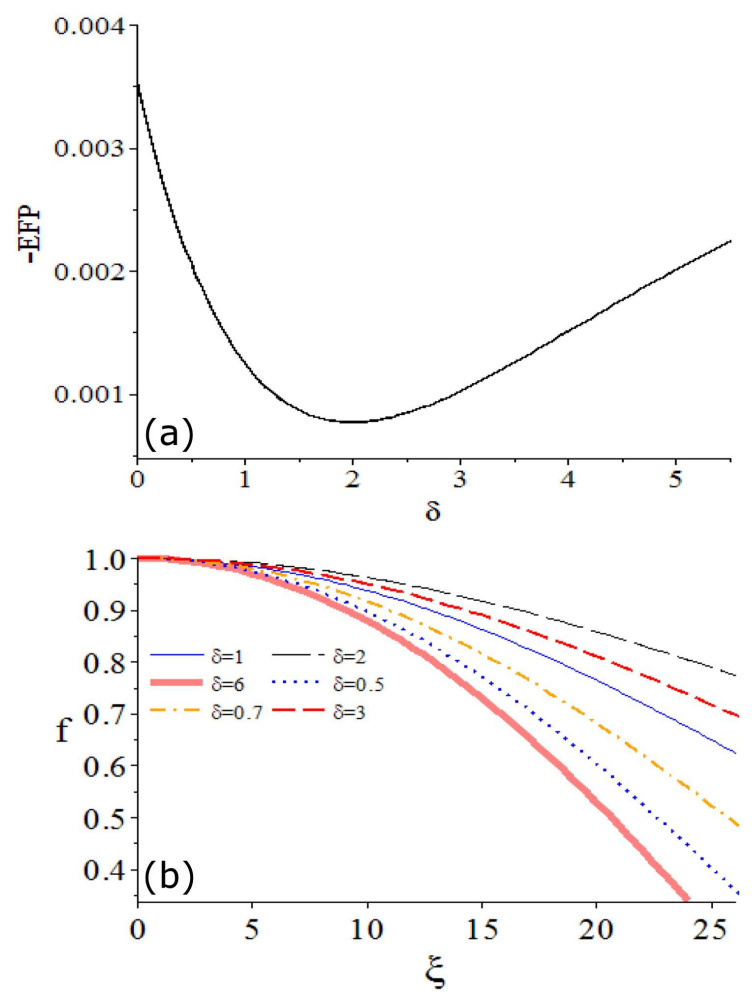

Fig. 2. (a) The introduced EFP parameter versus $\delta$. (b) The result of solving for self-focusing Eq. (9). 

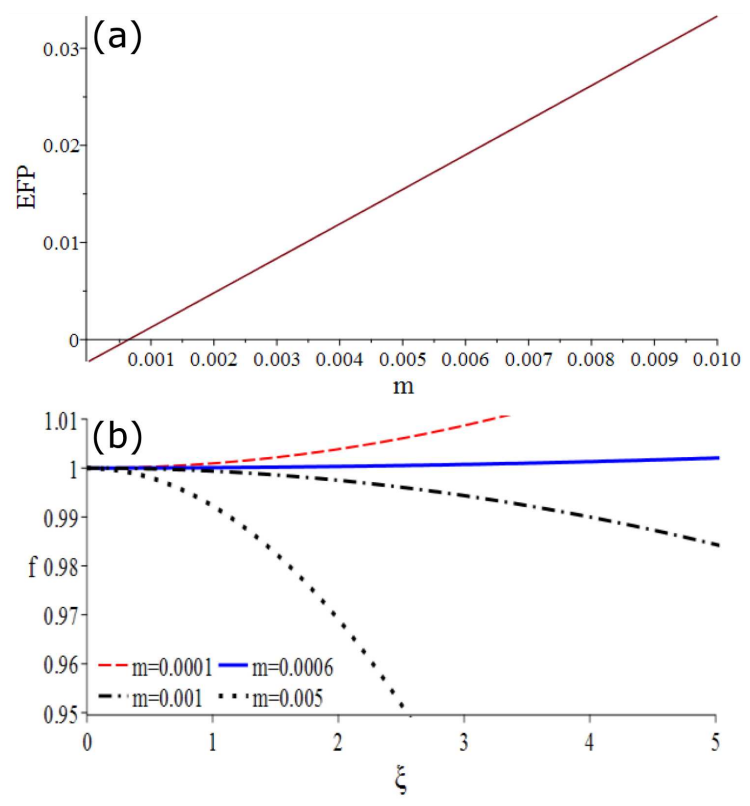

Fig. 3. (a) The introduced EFP parameter versus $m$.

(b) The result of solving for self-focusing Eq. (9).

- As is mentioned, the negative, positive, and zero value of EFP predicts the diverged, focused, and unchanged beam, respectively. Therefore, for $n<1$ and $n>7$, the negative EFP predicts the beam divergence and the positive EFP for $1<n<7$ predicts the focusing of beam.

- Moreover, discussing the EFP behavior gives the possibility for comparing the focusing of beam for different initial conditions. For instance, Fig. 1a shows the negative EFP for $n=0.5$ and $n=10$ with $\mathrm{EFP}_{n=0.5}<\mathrm{EFP}_{n=10}$. This predicts more divergence of beams with $n=0.5$ with respect to $n=10$.

The validity of the above mentioned results is verified in Fig. 1b, by solving for the boundary in Eq. (9), and the variation of beam width along the propagation length shows good conformity with the EFP behavior.

Figure 2a indicates the nonlinear EFP behavior versus $\delta$ parameter, which characterizes the position of the ring ripple on the wave front of the main beam. The inverse dependence of EFP at $\delta<2$ and the direct dependence of $\mathrm{EFP}$ on $\delta$ for $\delta>2$ with the minimum value of $\mathrm{EFP}$ at $\delta \approx 2$ is seen. Solving for the boundary in Eq. (9), this result is confirmed in Fig. $2 \mathrm{~b}$.

The effect of $m=\left(r_{1} / r_{0}\right)^{2}$, which represents the ratio of ripple width with respect to the initial beam width of the main beam $r_{1}$, is shown in Fig. 3a. This figure indicates the direct relation between EFP and $m$, which predicts the stronger focusing of the main beam for the rings of larger beam width. The results of solving for Eq. (9) for different $m$ are presented in Fig. 3b and good conformity with the EFP result is shown.
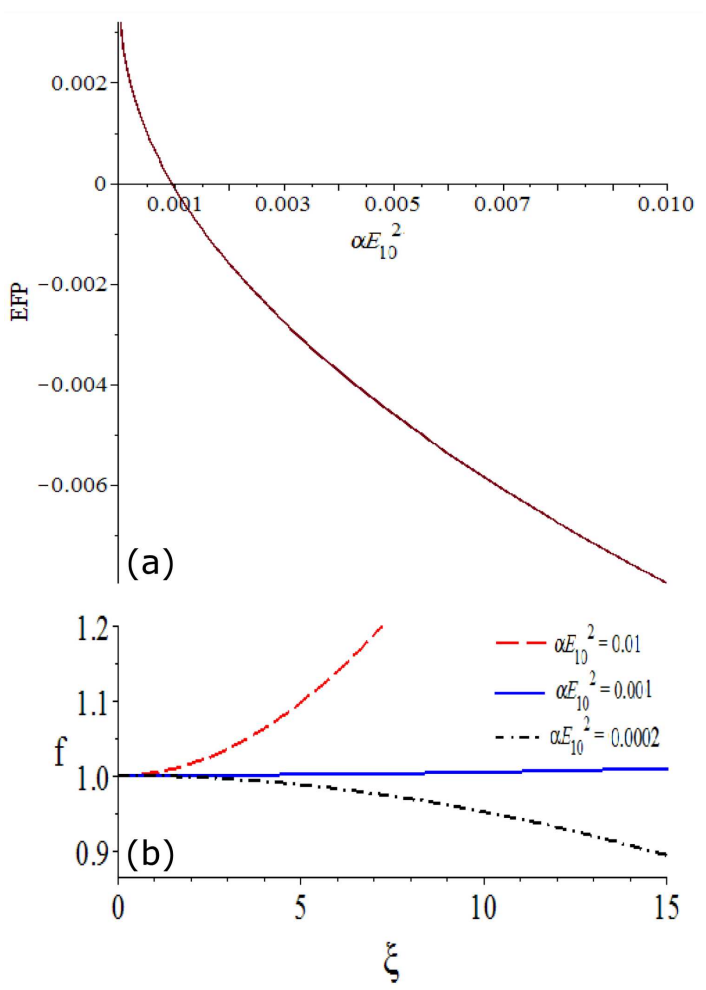

Fig. 4. (a) The introduced EFP parameter versus $\alpha E_{10}^{2}$. (b) The result of solving for self-focusing Eq. (9).
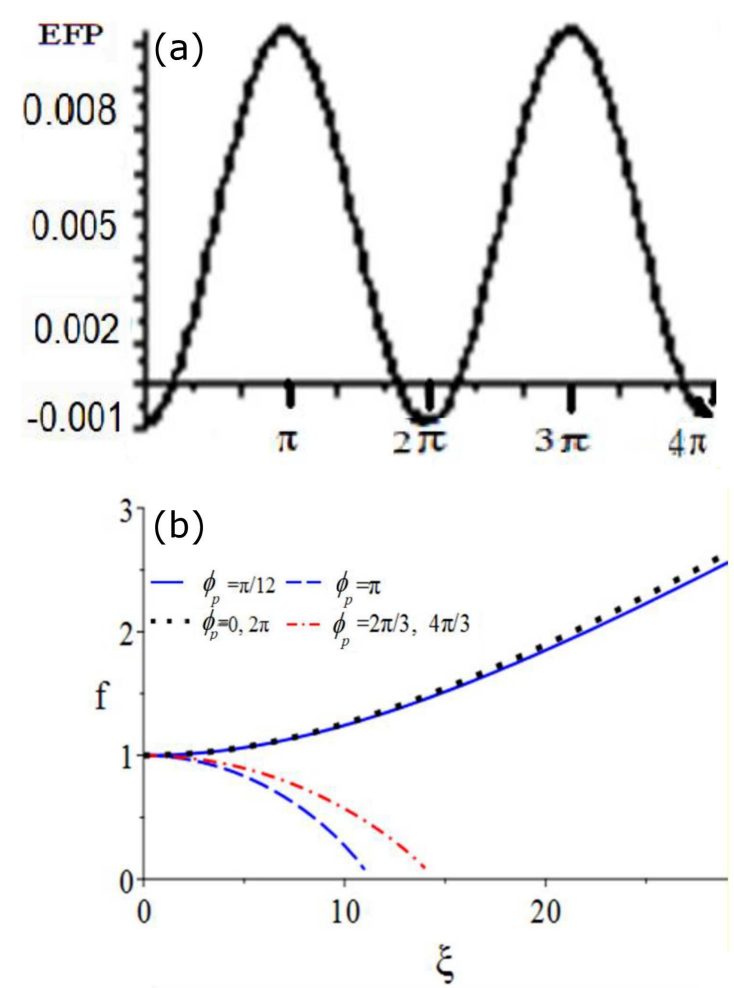

Fig. 5. (a) The introduced EFP parameter versus $\phi_{p}$. (b) The result of solving for self-focusing Eq. (9). 
The effect of the initial intensity of the ripple on selffocusing is studied in Fig. 4a, by plotting the introduced parameter of EFP versus $\alpha E_{10}^{2}$. This figure indicates the inverse dependence of EFP on $\alpha E_{10}^{2}$. It shows also that for the initial set of parameters, as Eq. (10) for $\alpha E_{10}^{2}<0.001$, the EFP is positive and therefore the beam will be focused. For $\alpha E_{10}^{2}=0.001$ the EFP parameter is zero, and therefore the beam size will not change. In turn, for $\alpha E_{10}^{2}>0.001$ the EFP parameter is negative. Therefore, the beam will be diverged. Solving for Eq. (9), this result is confirmed in Fig. $4 \mathrm{~b}$.

The effect of the initial phase difference between the Gaussian beam and the ring ripple $\phi_{p}$ on EFP is shown in Fig. 5a and the nonlinear dependence of EFP on $\phi_{p}$ is seen. The results, after solving Eq. (9), in Fig. 5b also indicates the good conformity with the EFP behavior.

\section{Conclusion}

Based on the introduced parameter of focusing in plasma, the nonlinear dependences of self-focusing on the ripple parameters are found. Regarding the initial standard set of parameters, the behavior of introduced parameter indicates the nonlinear dependences of selffocusing on the $n$ and $\delta$ (the parameters which describe the position of ripple on the wave front of the main beam) and $\phi_{p}$ (the initial phase difference between the main beam and the ring ripple). The direct dependence of self-focusing on the initial width of the ripple and the inverse dependence on the intensity of ripple are also found. The results of the beam width variation along the propagation axis confirm the obtained results.

\section{References}

[1] J. Badziak, S. Glowacz, H. Hora, S. Jablonski, J. Wolowski, Laser Part. Beams 24, 249 (2006).

[2] A. Pukhov, D. Brugge, I. Kostyukov, Plasma Phys. Control. Fusion 52, 124039 (2010).

[3] C. Thaury, F Quéré, Physica B At. Mol. Opt. 43, 213001 (2010).

[4] M. Shirozhan, M. Moshkelgosha, R. Sadighi-Bonabi, Laser Part. Beams 32, 285 (2014).

[5] W. P. Leemans, C. G. R. Geddes, J. Faure, C. Tóth, J. Tilborg, C.B. Schroeder, E. Esarey, G. Fubiani, Phys. Rev. Lett. 91, 074802 (2003).
[6] A. Akhmanov, A.P. Sukhorukov, R.V. Khokhlov, Sov. Phys. Usp. 10, 609 (1968).

[7] M. Moshkelgosha, R. Sadighi-Bonabi, IEEE Trans. Plasma Sci. 41, 1570 (2013).

[8] R. Sadighi-Bonabi, M. Moshkelgosha, Laser Part. Beams 29, 453 (2011).

[9] T.S. Gillandn, S. Saini, Laser Part. Beams 25, 283 (2007).

[10] R.K. Khanna, K. Baheti, Pramana J. Phys. 56, 755 (2006).

[11] H.D. Pandey, V.K. Tripathi, Phys. Fluids B 2, 1221 (1990).

[12] A. Sharma, M.P. Verma, G. Prakash, M.S. Sodha, J. Appl. Phys. 95, 2963 (2004).

[13] M.S. Sodha, J.K. Sharma, D.P. Tewari, R.P. Sharma, S.C. Kaushik, J. Appl. Phys. 50, 6214 (1979).

[14] P. Rawat, R. Gauniyal, G. Purohit, Phys. Plasmas 21, 062109 (2014)

[15] M.S. Sodha, S. Misra, S.K. Mishra, Laser Part. Beams 27, 689 (2009).

[16] M.S. Sodha, A. Sharma, G. Prakash, M.P. Verma, Phys. Plasmas 11, 3023 (2004).

[17] M.S. Sodha, D.P. Singh, R.P. Sharma, Appl. Phys. 18, 97 (1979).

[18] M.S. Sodha, A. Sharma, Phys. Plasmas 14, 044501 (2007).

[19] S. Misra, S.K. Mishra, Phys. Plasmas 15, 0923071 (2008).

[20] S. Misra, S.K. Mishra, J. Plasma Phys. 75, 545 (2009).

[21] M.S. Sodha, S. Misra, S.K. Mishra, Laser Part. Beams 27, 689 (2009).

[22] H.S. Brandi, C. Manus, G. Mainfray, T. Lehner, G. Bonnaud, R. Phys. Fluids 5, 3539 (1993).

[23] F. Osman, R. Castillo, H. Hora, Plasma Phys. 61 263 (1999).

[24] M.S. Sodha, S. Konara, K.P. Maheshwari, J. Plasma Phys. 48, 107 (1992).

[25] M.S. Sodha, A.K. Ghatak, V.K. Tripathi, Self Focusing of Laser Beams in Dielectrics, Plasmas and Semiconductors, Tata-McGraw-Hill, New Delhi 1974.

[26] M.S. Sodha, A.K. Ghatak, V.K. Tripathi, Prog. Opt. 13, 169 (1976).

[27] M. Moshkelgosha, IEEE Trans. Plasma Sci. 44, 6 (2016). 